\title{
2006-1843: SPREADSHEET INSTRUCTION WITHIN A FIRST YEAR CHEMICAL ENGINEERING COURSE
}

\section{William Josephson, Tuskegee University}

William Josephson is a Research Associate Professor in Tuskegee University's Chemical Engineering Department. His research interests lie in the pulp \& paper industry and in engineering education at both the secondary school and university levels.

\section{Jaya Krishnagopalan, Tuskegee University}

Jaya Krishnagopalan is a Professor in Tuskegee University's Chemical Engineering Department. She is the long-time teacher of the department's Material \& Energy Balances course.

\section{Nader Vahdat, Tuskegee University}

Nader Vahdat is the Department Head of Tuskegee University's Chemical Engineering Department. His research interests include adsorption, permeation of chemicals through polymeric materials, membrane separation and fire extinguishing agents. 


\title{
Spreadsheet Instruction Within A First Year Chemical Engineering Course
}

\begin{abstract}
This paper reports upon our experiences with incorporating formal instruction in spreadsheet software (Microsoft ${ }^{\circledR}$ Excel) in our department's introductory chemical engineering course. Spreadsheet instruction was conducted in the department's computer laboratory with all the students able to run Excel during the class session. Instruction started with spreadsheet basics and over the course of the semester introduced a number of numerical techniques that can be implemented in a spreadsheet environment.

The use of "real-world" chemical engineering examples to provide instruction in various mathematical techniques and their implementation in the Excel environment is described. A third-order expression for heat capacity was used to illustrate the concept of trial-and-error searches. Chemical reactor design equations were given to the first year students along with a graphical interpretation of the equations; the trapezoidal rule was used within Excel in order to compute reactor volumes. Excel's iteration capabilities were illustrated both by simulations of chemical processing systems with a recycle stream and by two-dimensional heat conduction simulations.
\end{abstract}

\section{Introduction}

In 2003 the Chemical Engineering Department of Tuskegee University engaged in a self-study to examine the status of computer science education within the curriculum ${ }^{1}$. One of the recommendations of that study was that formal instruction in spreadsheet software (Microsoft ${ }^{\circledR}$ Excel) be incorporated in the department's introductory chemical engineering course (CENG 110). That recommendation was adopted and this paper reports on our experiences to date.

The roster in CENG 110 is usually composed almost entirely of first year students; the course has no pre-requisites and is actually open to all majors (although during the period reported on all CENG 110 students were chemical engineers). The course meets once a week for a two hour session. Typically the Excel portion of class was taught during the second hour of class. Spreadsheet instruction was conducted in the department's computer laboratory with all the students able to run Excel during the class session.

Some aspects of Excel had previously been taught by one of the authors (WJ) to select first year engineering students. These first year students were part of Tuskegee's FASTREC (Freshman Accelerated Startup and Training for Retention in Engineering Curricula) program, an 8 week summer program in which incoming T.U. freshmen take introductory engineering and mathematics courses. FASTREC students typically are aerospace, chemical, electrical or mechanical engineering majors. When teaching Excel as part of CENG 110, however, a concerted effort was made to incorporate examples and problems specific to chemical engineering. 
Although the examples presented in this paper pertain to chemical engineering, they certainly can be applied to any engineering field. For example, this paper's iteration example (presented under the heading Trial \& Error) is from our chemical engineering thermodynamics courses. But iteration is a concept important to all engineering disciplines and examples abound of situations in other fields in which an iterative method is best suited for problem solution; e.g., determining the height in a rectangular channel given volumetric flowrate and geometry information using the Chezy formula ${ }^{2}$ or determining an unknown current in a non-linear DC circuit ${ }^{3}$.

Our overall objective in teaching Excel to our first year students is to provide instruction in various mathematical techniques and methods of thinking as well as to introduce the first year student to a tool that they will use throughout their stay at Tuskegee and, most probably, for a good number of years thereafter. Most if not all of the techniques taught should be applicable to non-Excel situations (e.g., a lone student with a calculator should be able to utilize knowledge gained from the course to solve realistic problems). The use of realistic chemical engineering problems was deemed important as it gave the students a taste of things to come.

This paper reports on some of the lessons learned (and still being learned) as the CENG 110 students were given instruction in Excel. First we report on the basic aspects of Excel that we have found necessary to teach. Then a series of example problems is presented; for each example we report on the general goal that we are trying to achieve and we report on the specific aspects of Excel that we utilize to achieve that goal.

\section{The Basics}

We have found it necessary to discuss the most fundamental aspects of Excel with first year students regardless of their professed level of familiarity with Excel. There is usually at least one student who, even though he/she doesn't admit it, has never used the software.

The discussion will start off with an overview of spreadsheet software: e.g., a "spreadsheet" refers to a 2-dimensional matrix of cells, the cells may contain static data such as alphabetic characters or numbers, etc. The cell referencing system is covered: individual cells are referred to with a column-row nomenclature and blocks of contiguous cells can also be referred to (i.e., cell ranges). Especially in the introductory sessions an emphasis is made on keeping presentations simple and not getting bogged down in advanced topics. Thus, the 3-dimensional aspects of Excel are not covered, nor are macros, database manipulations, etc.

The power of Excel and other spreadsheet software lies in the ability to insert formulas in cells which dynamically reference other cells. Proper formula nomenclature (i.e., use of the $=\operatorname{sign}$ ) is extensively covered along with the hierarchy of mathematical operators.

The last critical basic concept that the students learn has to do with the copying of cells with formulas in them. Cell references in formulas are relative by default and when copying formulas any embedded cell references will be updated to take into account the formula's new location. A great many things can be done once the students are understanding of this idea. A quick way to determine if the students are appreciative of the concept is to ask them to generate a column of 
numbers in series, e.g., from 1 to 100 . Students unclear on the concept will simply manually enter in the numbers (or start to, before they realize that there must be an easier way!) while those who have learned how to make use of relative references will quickly determine that the best course of action is to copy a formula that generates a value of 1 more than that of the data in the preceding cell.

Finally the students are taught advanced cell reference techniques, namely the use of absolute references. In Excel this is done by using the \$ character in the cell reference; e.g., \$D\$3 is an absolute reference and, if embedded in a copied formula, any new formula will still refer to the cell in column D and row 3. The use of cell names as shorthand for absolute cell references may also be taught.

\section{Graphing}

The first and primary use of Excel for many of our students is in data presentation, not data generation. In the past students have learned about and used the data plotting aspect of Excel largely on their own. This was unfortunate as Excel is, at heart, a software package designed for business applications and sometimes default actions can lead scientific/engineering users astray. Such was often the case when our self-taught students would attempt to use Excel to plot a set of $x-y$ data. The correct type of Excel chart to use for $x-y$ data is the "XY (Scatter)" chart. However, many students elect to use Excel's "Line" chart to plot their $x-y$ data. The students see the preview of what a line chart looks like and figure that its form (data points connected by straight lines) is what they want. However, Excel's Line Chart does not display x-y data sets as numerically related items. When utilizing a Line Chart any $\mathrm{x}$ data is treated as the name of a category. $\mathrm{Y}$ data is still treated as a numerical entity but any numerical relationship between the two is lost and is not displayed on the plot. Figures 1 and 2 illustrate the problem. In both figures a data set of $\{(1,12),(2,20),(4,25),(6,50)\}$ has been entered. In Figure 1 a line chart has been used to display the data. Note that the $\mathrm{x}$ axis does not have a numerical component. For example, the horizontal distance from 1 to 2 on the $\mathrm{x}$ axis is equal to the horizontal distance from 2 to 4 . Figure 2, in which an xy scatter chart has been used, shows the correct representation of the data. The difference between the two can be subtle, as it is here, when the change in $\mathrm{x}$ data increment is not great. 


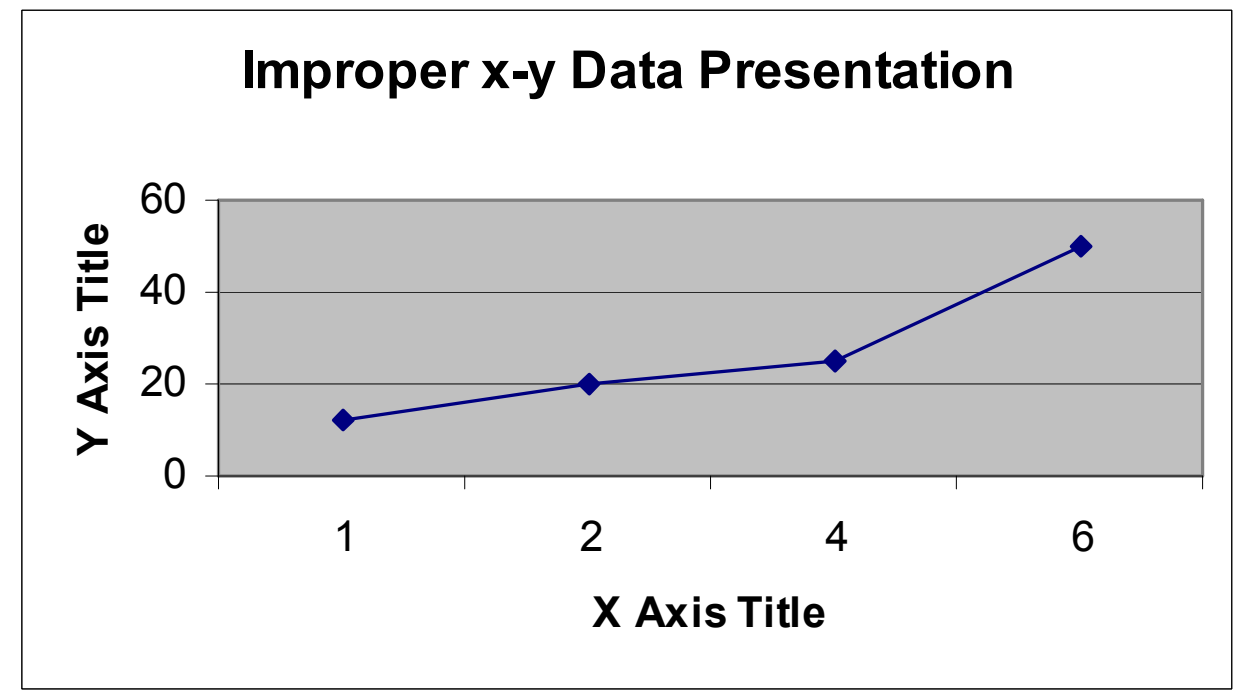

Figure 1 - Use of A Line Chart

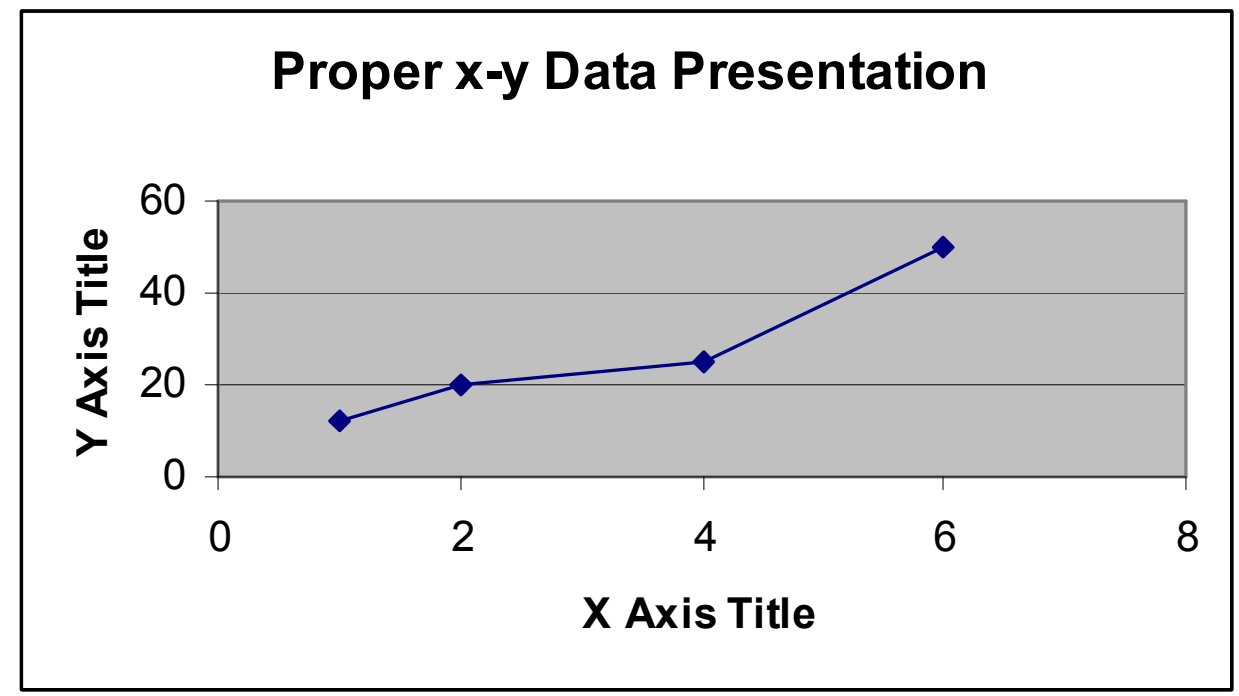

Figure 2 - Use of An X-Y Scatter Chart

Excel's generation of trendlines is another aspect of graphing that is useful for the first year students. The students are first taught why engineers often seek to generate and use an equation that approximates a set of xy data. They are then given instruction in the method of least squares. Using the appropriate formulas each student demonstrates an ability to generate a linear equation that best fits a set of data. It is important to note that the students do this outside of Excel using only their calculators. Only after the students have learned how to generate a linear equation on their own are they shown how the task can be simplified using Excel's trendline feature.

Trail \& Error 
During the instruction of Excel basics the students are taught applications and techniques. One of the most useful techniques for first year students is the use of iteration in instances where an equation is not explicit in an unknown variable. Such situations are common in chemical engineering courses starting with the sophomore year. Figure 3 illustrates a case from thermodynamics ${ }^{4}$ in which a given equation relates $\tau$, a dimensionless variable equal to a temperature divided by the initial temperature $T_{0}$, to other parameters such as heat transfer $Q$, the gas constant $\mathrm{R}$, the initial temperature $\mathrm{T}_{\mathrm{o}}$, molar amount $\mathrm{n}$ and a heat capacity relationship dependent on the material (parameters A, B, C and D).

$$
\mathrm{Q}=\mathrm{nR}\left[\mathrm{AT}_{\mathrm{o}}(\tau-1)+\mathrm{BT}_{\mathrm{o}}^{2}\left(\tau^{2}-1\right) / 2+\mathrm{CT}_{\mathrm{o}}^{3}\left(\tau^{3}-1\right) / 3+\mathrm{D}(\tau-1) / \tau \mathrm{T}_{\mathrm{o}}\right]
$$

As the equation is cubic in $\tau$ it is not able to be solved analytically by first year students. However, an iterative approach (or "trial and error" in student-speak) does provide a relatively quick path to the correct value of $\tau$. The students are instructed to simply guess a value of the unknown variable $\tau$, evaluate the expression, determine the resulting error, re-estimate $\tau$ and repeat until the generated error is at an accepted minimal level. When introducing this method it is recommended to the students that the equation be rearranged so that one side of it will be equal to zero:

$$
\mathrm{f}(\tau)=\mathrm{AT}_{\mathrm{o}}(\tau-1)+\mathrm{BT}_{\mathrm{o}}^{2}\left(\tau^{2}-1\right) / 2+\mathrm{CT}_{\mathrm{o}}^{3}\left(\tau^{3}-1\right) / 3+\mathrm{D}(\tau-1) / \tau \mathrm{T}_{\mathrm{o}}-\mathrm{Q} / \mathrm{nR}=0
$$

The goal of the student is then to find a value of $\tau$ for which the function equals zero. Zero is chosen because it is easy to determine when two successive iterations bound the answer: the resultant functions will be of differing signs. This is of obvious use when determining the value of the next estimate. It is important to note that the trial and error technique is first taught outside of the Excel setting. As is the case with the method of least squares discussed above, all students are capable of using trial and error using only their calculators. However, the use of Excel does allow for the easy application of the method in a variety of situations. The problem shown in Figure 3 illustrates many aspects of Excel discussed up to this point: the spreadsheet user has made multiple copies of a formula with both relative and absolute references in it (the $\mathrm{f}(\mathrm{Tau})$ formula), application of the formula itself requires a careful eye in terms of proper use of parentheses, etc. Note also the use of cell names when possible instead of harder-to-interpret absolute cell references. Cell B4, for instance, is also named "To" (signifying the original temperature $\mathrm{T}_{\mathrm{o}}$ ) and it is this name which is referred to where appropriate in any formulas.

Semi-advanced users may realize at this point that Excel itself offers built-in methods to determine values of variables such as $\tau$ above. The "Goal Seek" tool, for instance, allows for the automatic manipulation of one cell's contents in order to change another cell's contents to a given value. For the example shown in Figure 3 this would simply entail instructing Excel to set a cell in the $\mathrm{f}(\mathrm{Tau})$ column to zero by changing the value of the cell immediately to its left. Excel would then adjust and re-adjust the Tau variable until the given condition was met. While such an approach is obviously a great saver of time, it also obviously does nothing towards educating the students in the how and why of the trial and error technique. Accordingly, although we do instruct our students in certain advanced methods such as the Goal Seek tool, we do so only after the basic technique has been mastered. 


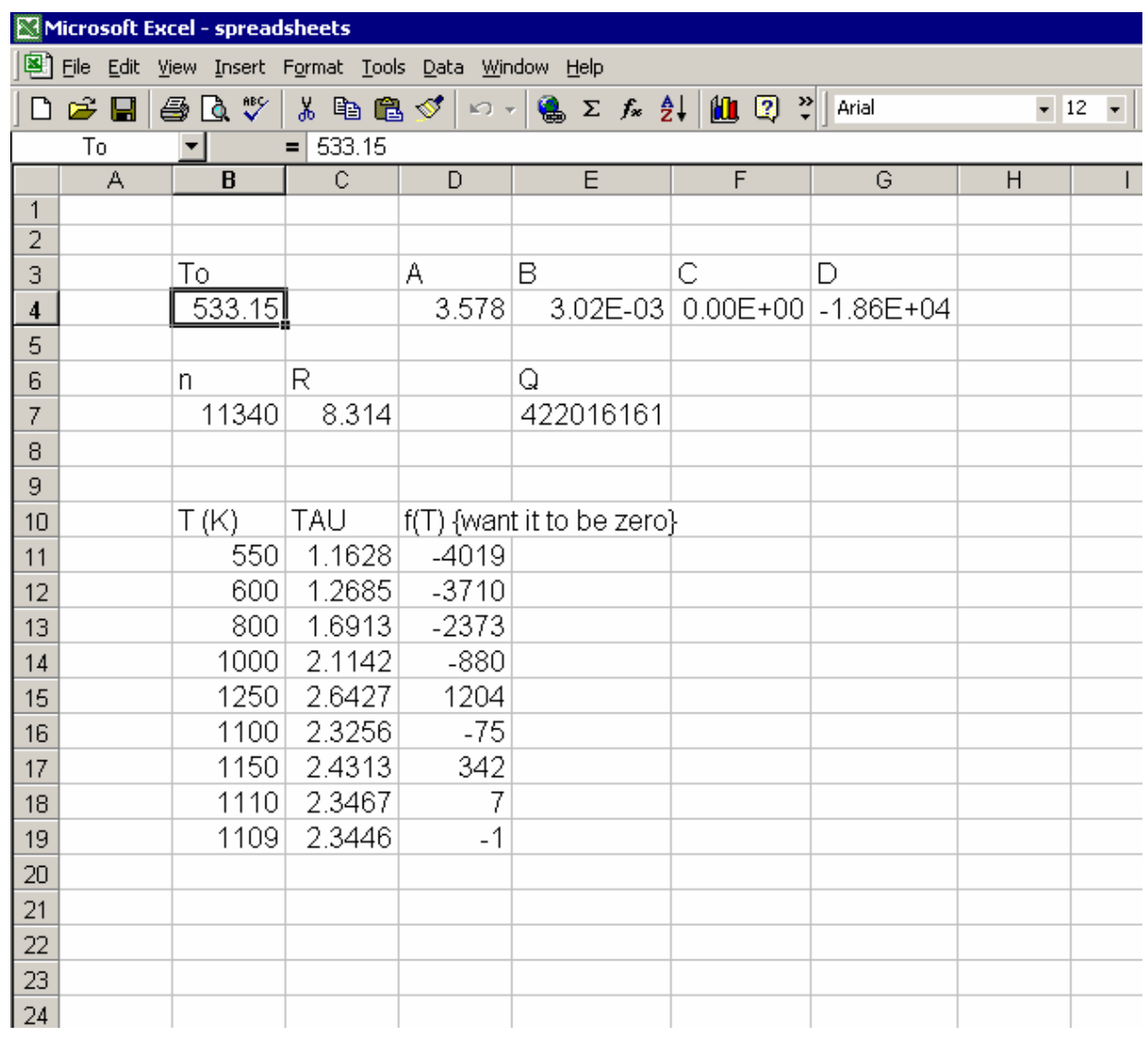

Figure 3 - Trial \& Error to Find the Unknown Tau

\section{Trapezoidal Rule}

The trapezoidal rule is another relatively simple procedure which we have found to be useful when giving instruction in Excel. There are many occasions to use the trapezoidal rule or other methods of numerical integration in the chemical engineering curriculum. Figure 4 shows an application from our chemical reaction engineering course ${ }^{5}$. In this example the first year students were given a set of $x-y$ data corresponding to fractional conversion of a reactant ( $x$ values) and the reciprocal reaction rate (y values). They are told that the reactor volume needed to achieve a given conversion was directly related to the area underneath the conversion vs reciprocal reaction rate curve (from conversion equals zero to the desired value). After some basic instruction in the use of the trapezoidal rule (e.g., area of a trapezoid equals its base times the average of the two heights) the students were able to construct spreadsheets in which the desired area was calculated. Note that in Figure 4 the given data is plotted on an xy scatter chart; although such a plot is useful for problem visualization it is not necessary for successful calculation of the area underneath the curve. 


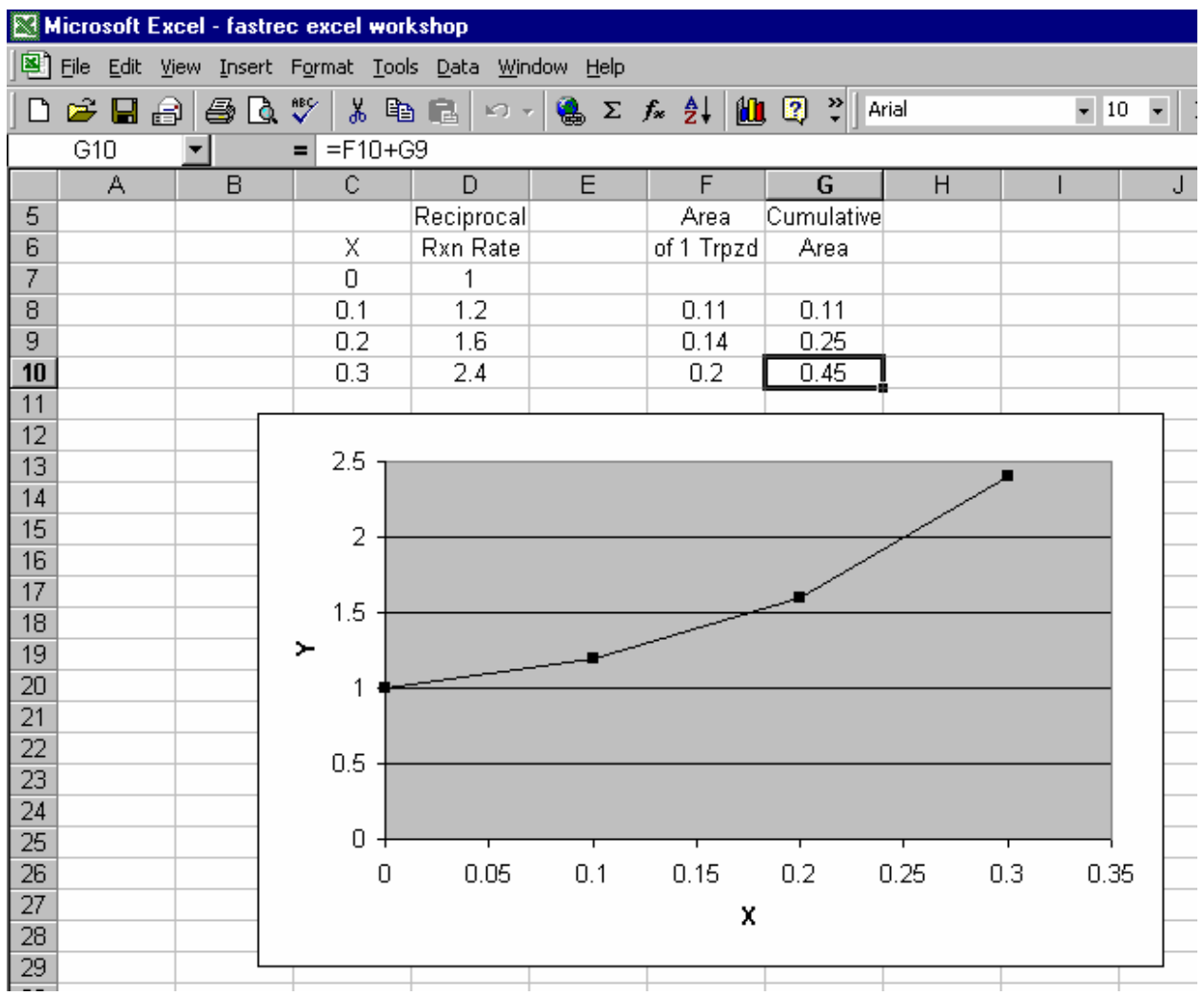

Figure 4 - Using the Trapezoidal Rule

\section{Differential Relationships I}

The use of mathematical toolkit software to model differential relationships is common in the advanced courses in the chemical engineering curriculum. Excel can also be used for this purpose and is often more illustrative of the concepts involved. However, instructing first year students in some of these applications can be somewhat tricky since not all of the students have taken an introductory calculus course and thus do not know what a derivative is. We have attempted to instruct the students using different types of example problems and have found that it is difficult to convey the relevant concepts using real world chemical engineering situations. In Figure 5, for example, we are given a set of time and concentration (of chemical A) data. We wish to generate the derivative of concentration; i.e., $\mathrm{dC}_{\mathrm{a}} / \mathrm{dt}$. $\mathrm{dCa} / \mathrm{dt}$ can be approximated by delta $\mathrm{Ca} /$ delta $\mathrm{t}$; this is done in column $\mathrm{J}$ of Figure 5 . A plot showing how the derivative changes with concentration is given as Figure 6. Note that the concentration data on the $\mathrm{x}$ axis is plotted in reverse order so as to mimic chronological direction (i.e., the concentration decreases as time increases).

While the first year students are all capable of following the 'recipe' for approximating the derivative, they do not have enough experience to have a feel for what is really going on. In particular, the students have little understanding of the necessity of generating a new set of data (concentration data, for instance) if one wants to plot the derivative versus concentration or time. Although the students can memorize the "how" (e.g., you must match up delta $\mathrm{Ca} /$ delta time data with the average of the two concentrations used) they don't understand the "why" (e.g., the 
delta $\mathrm{Ca} /$ delta time data is generated using two concentrations and so therefore it's best thought of as being most valid "in the middle" of the two concentrations used).

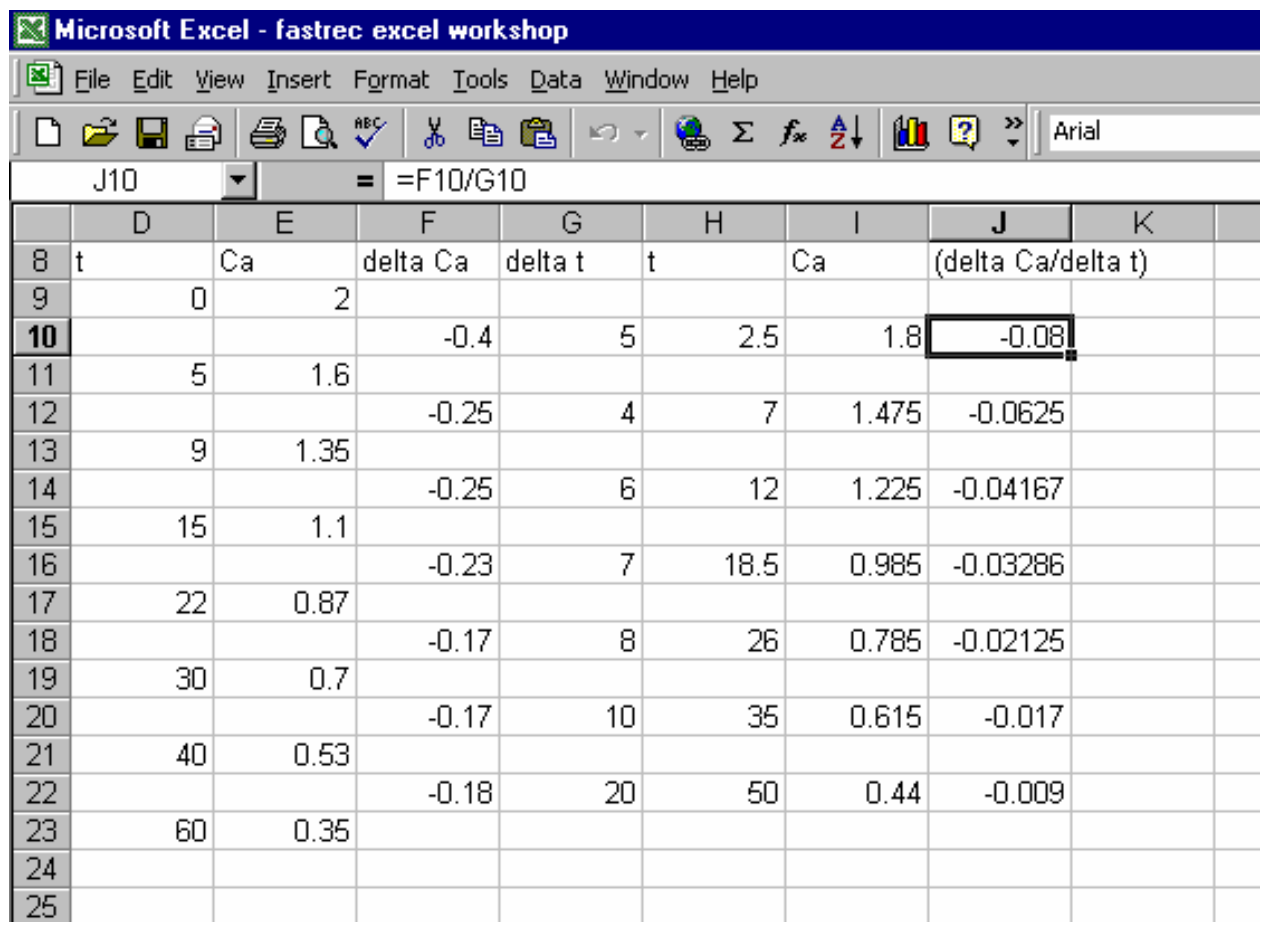

Figure 5 - Modeling a Differential Relationship

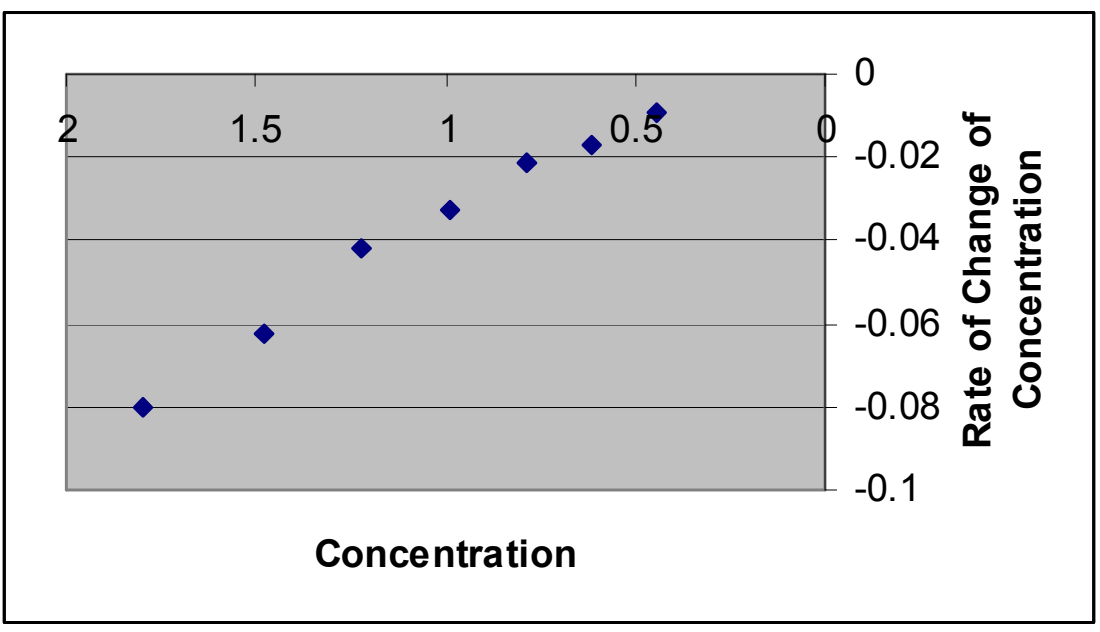

Figure 6 - Plotting dCa/dt versus Ca

The first year students are capable of understanding the basic concepts behind differential relationships when presented with examples more within the realm of their experiences. Figure 7 shows a data set documenting distance traveled by car and elapsed time. First year students understand that the change in distance traveled divided by the change in time is simply velocity. They also understand why a given calculated velocity must be matched with distances or time 
data not in the original data set. For example, when asked at what time is the car traveling at 40 miles/hour (the first calculated velocity), most students readily comprehend that at time zero the car is just starting out and at time 0.1 hours the car must be going faster than $40 \mathrm{mph}$. Therefore, the car is going exactly $40 \mathrm{mph}$ somewhere in between 0 and 0.1 hours. The average of the two times serves as a reasonable approximation.

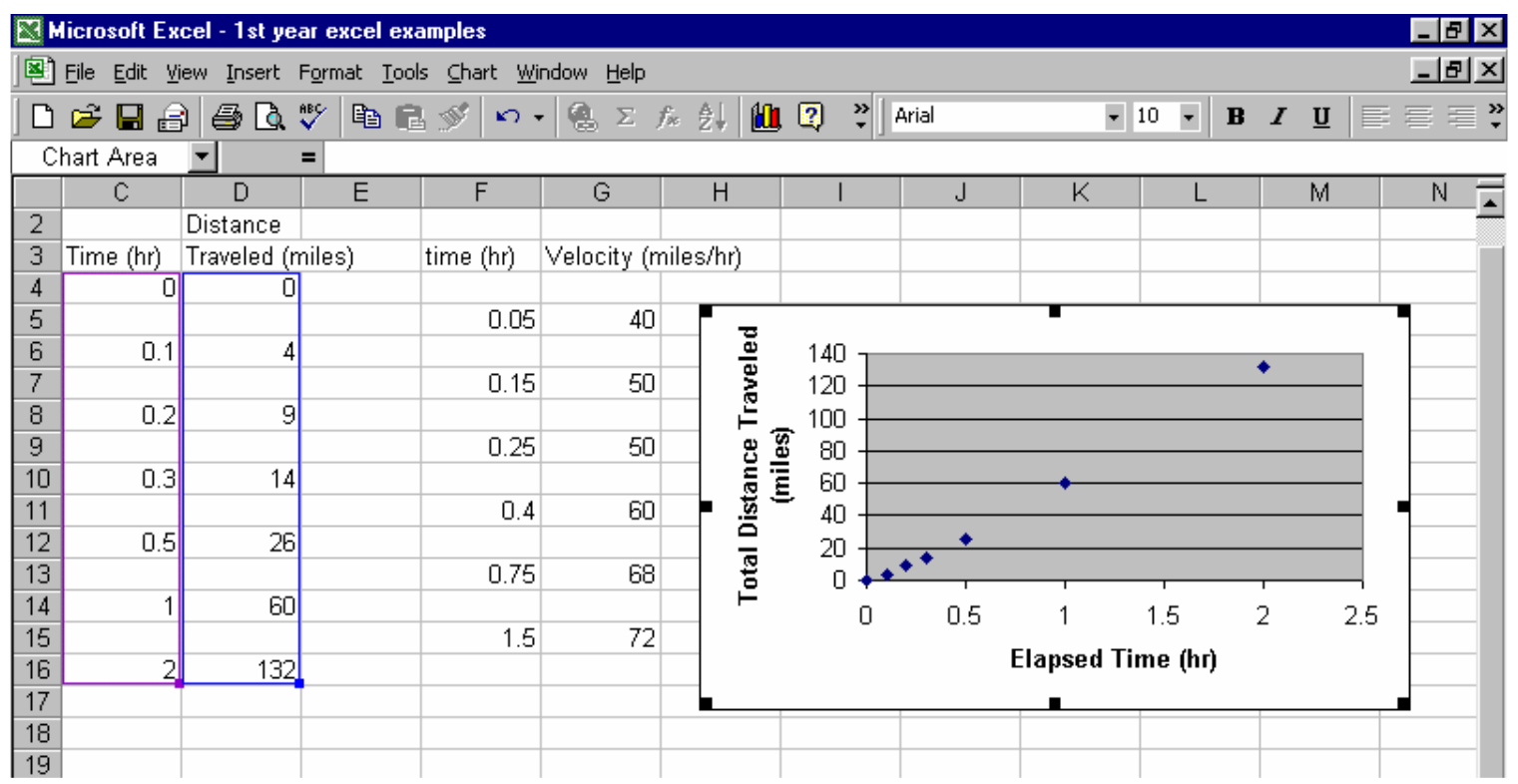

Figure 7 - A non-Chem Eng Differential Relationship

\section{Differential Relationships II}

Given the difficulties in using differential relationship examples discussed above it is not surprising that more complex situations are mentioned only fleetingly in the first year course. Figure 8 illustrates the outcome of an analysis of a plug flow reactor packed with a catalyst ${ }^{6}$. It can be shown that two linked ordinary differential equations describe the reactor:

$$
d X / d W=f(X, P) \& d P / d W=g(X, P)
$$

where $\mathrm{W}$ is the weight of the catalyst, $\mathrm{X}$ is the fractional conversion of a fed reactant and $\mathrm{P}$ is the reactor pressure. Spreadsheet simulation of the reactor can be successful if one uses an approach based on Euler's method ${ }^{7}$ : treat $\mathrm{W}$, the amount of catalyst, as an independent variable, utilize initial values of $\mathrm{X}$ and $\mathrm{P}$ ( 0 and 10 , respectively, in this case), evaluate the differentials and use them to generate new values of $\mathrm{X}$ and $\mathrm{P}$. 


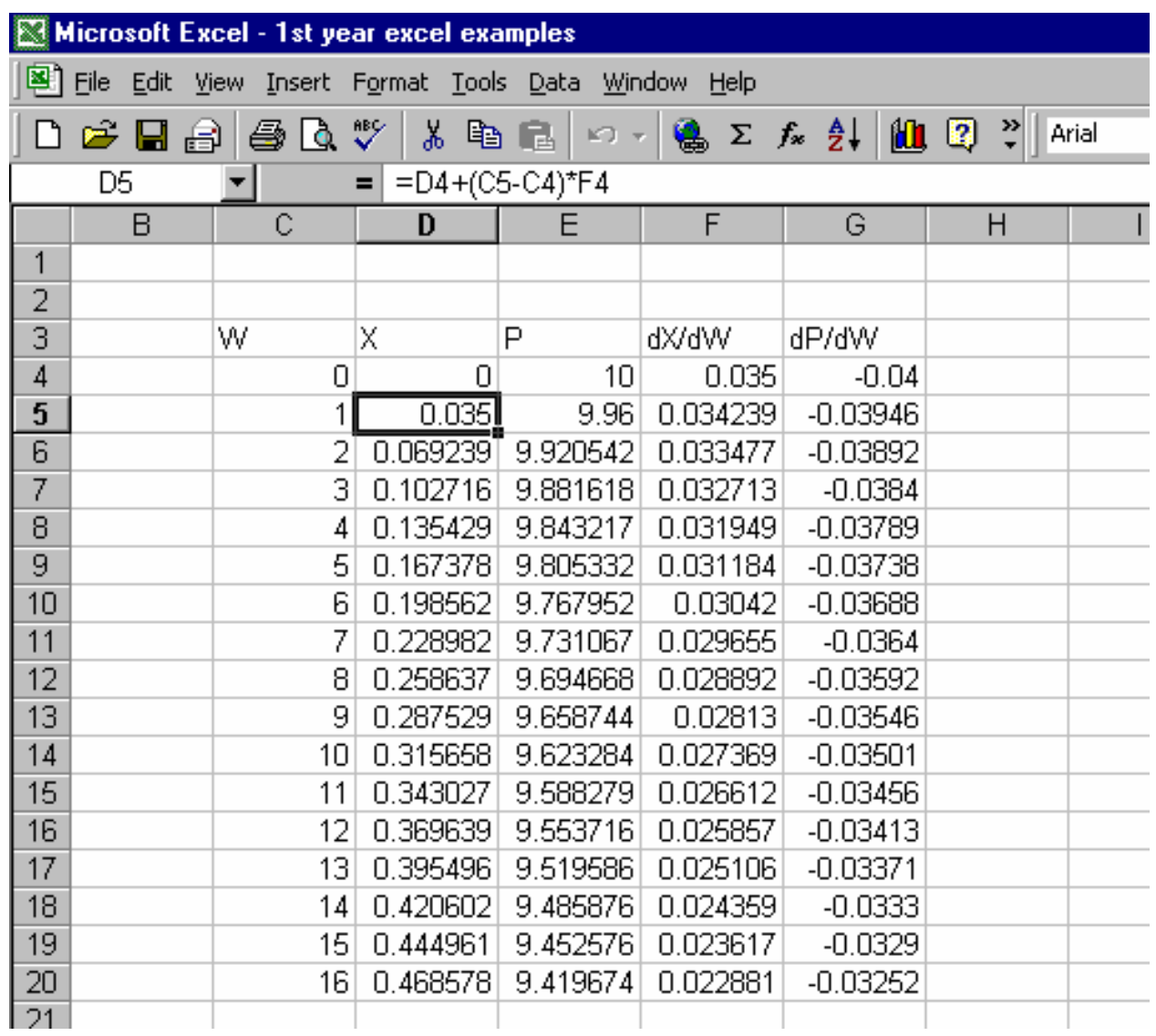

Figure 8 - Modeling a Reactor Described by 2 o.d.e.s

Although the above example is too complex for an in-depth analysis by first year students it is suitable for a brief overview by the students and does give the students an indication of the type of problems they will encounter in upper level courses within the chemical engineering curriculum.

\section{Use of Circular References I}

The problems associated with the above examples, while solved using Excel, could have been approached and solved using nothing more complicated than a hand calculator. However, the efficient solution of two types of chemical engineering problems requires the use of some sort of software package. The first type of problem is encountered whenever a system of unit operations has an internal recycle stream. In Figure 9, for example, the overall system consists of a 2 input/1 output mixing unit followed by a 1 input / 2 output operation which splits the input in half and apportions each half to one of the outputs. The overall system input consists of a single stream representing an input flow of 100 gallon/minute (gpm). This stream is one of the inputs to the mixing operation. The overall system output is one of the output streams of the splitting operation. The splitter's other output is recycled and is the other input to the mixing operation. This rather artificial example of a system with an internal recycle stream can be analyzed using basic chemical engineering balance techniques ${ }^{8}$ (e.g., an overall system balance shows that the system output equals the system input equals $100 \mathrm{gpm}$, the 50/50 split achieved in the splitting operation means that the recycle stream has the same flowrate as the system output stream and is thus $100 \mathrm{gpm}$, etc.). Despite its simplicity, however, modeling of the situation using Excel 
requires some specialized knowledge. The difficulty lies in the calculation of the mixing operation's output: it is obviously dependent on the value of the recycle stream but the recycle stream's value is (via the splitter) dependent on the value of the mixer output. When the relevant formulas are entered into a spreadsheet Excel will alert the user to this "circular reference" situation. The typical first year student (and a good many upper level students as well) is then stuck. The situation is resolved, however, if the user turns on Excel's automatic iteration feature (located in the Tools/Options/Calculation sub-menu). This allows Excel to resolve circular references by repeatedly calculating all formulas in the spreadsheet until minimal differences are detected from one iteration to the next. For the situation in Figure 9 the only flowrate data entered by the user was the system input of $100 \mathrm{gpm}$. During Excel's first attempt at resolving the circular reference a value of zero was used for the recycle stream flowrate. This resulted in a mixer output of 100 gpm which served as splitter input. Excel then calculated the recycle stream's value as $50 \mathrm{gpm}$ (based on the splitter's 50/50 split). Since this was in disagreement with the previous recycle stream value of zero gpm Excel recalculated all formulas using a recycle stream value of $50 \mathrm{gpm}$. This resulted in a mixer output of $150 \mathrm{gpm}$, which resulted in a recycle stream value of $75 \mathrm{gpm}$ and so on. Figure 9 shows the end result of Excel's calculations: a recycle stream value of essentially $100 \mathrm{gpm}$.

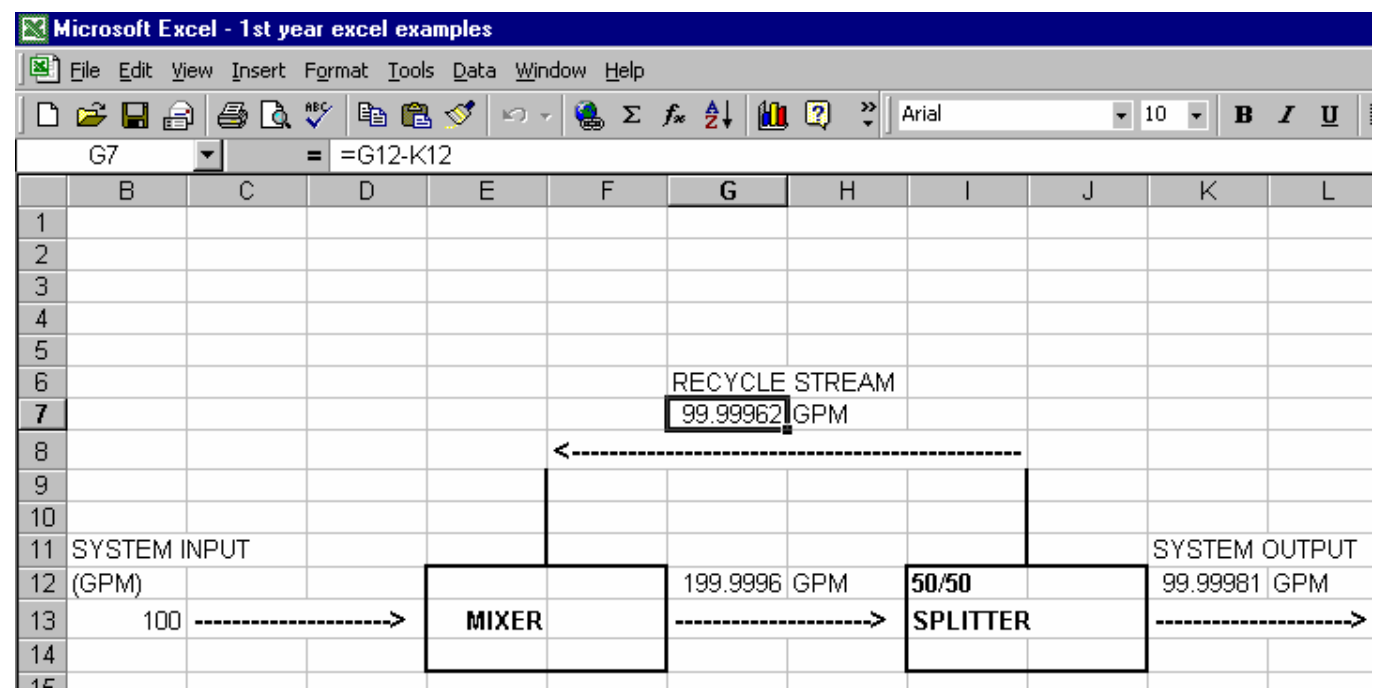

Figure 9 - Excel's Iteration Feature is Needed When Dealing with Circular References

This type of example is simple enough to be readily understood by the first year students and serves as an introduction to the principles behind the steady state simulation software such as Aspen Plus ${ }^{\circledR}$ that they will use in upper level courses. Other examples can be utilized when teaching the concepts to a mixed group of first year engineering students (e.g., FASTREC students). For instance, an electrical circuit diagram with amps taking the place of gallons per minute serves just as well as the above example. The key is that the students are introduced to circular references and how Excel can be instructed to deal with them.

\section{Use of Circular References II}

The final example presented here represents a different chemical engineering application but the same aspect of Excel. Figure 10 shows a spreadsheet representation of a 2-dimensional heat 
transfer problem ${ }^{9}$. A $8 \mathrm{~cm} \times 8 \mathrm{~cm}$ plate has its edges maintained at constant temperatures. The North and West edges are at $100^{\circ} \mathrm{C}$, the South edge is at $50^{\circ} \mathrm{C}$ and the East edge is at $75^{\circ} \mathrm{C}$. The students are asked to develop a map of the plate temperatures. The only other information given to the students is that the temperature at any point in the plate will be the average of the temperatures around that point. Heat capacity information is not needed as long as the plate is homogeneous.

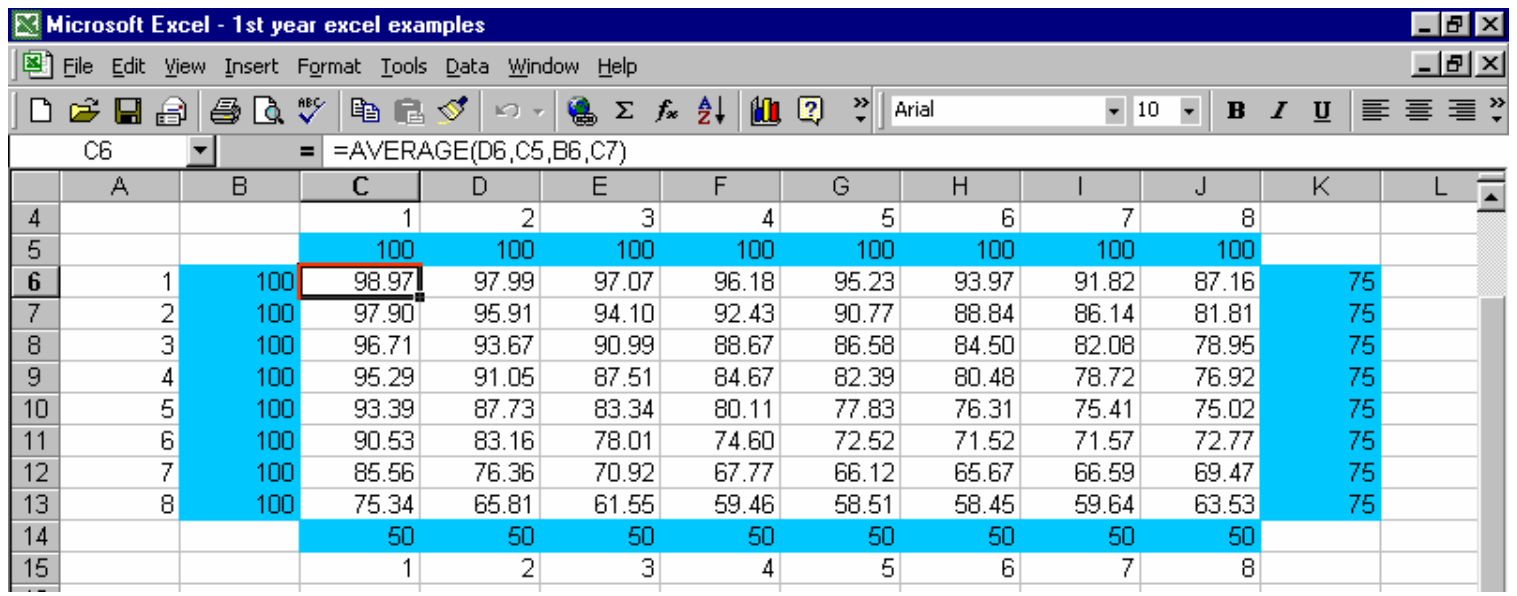

Figure 10 - Mapping Temperatures of a 2-D Plate

Coming on the heels of the first presentation involving circular references, most first year students quickly figure out that the way to develop the temperature map is to section the plate into nodes and utilize a formula that calculates the node temperature using the average of the surrounding temperatures. The formula for cell C6 in the figure shows how this is done. Note also the use of a different background color to distinguish the edges from the plate interior. This particular problem is actually rather fun to do primarily because it's computationally complex enough such that the students can watch the data change as Excel goes through its iterations.

\section{Conclusions}

The results from our instructional efforts in the first year course have been encouraging. The students are enthusiastic about the opportunity to gain a new and valuable skill set within the context of the introductory course. The time investment by the department is relatively small while the potential impact on subsequent courses is large.

We as instructors have learned some lessons during the administration of the course. Some lessons are obvious, others not so. As discussed above, we have found it necessary to go over the basics of Excel no matter the professed level of proficiency with the software. There is room for instructional improvement in this area; while we were prepared to discuss cell referencing, formula nomenclature and the like, quite frankly we were initially surprised to come across the occasional student who simply "didn't get it". As an example, such a student might, when asked to generate some values of the function $\mathrm{x}^{2}$, reply with "you write a formula like $=\mathrm{x}^{\wedge} 2$ " (i.e., the student didn't realize that " $x$ " means nothing to Excel and a cell reference should be used instead). 
Other aspects of instruction went as expected. The instructors knew going in to the course that graphing skills were critical in later engineering courses and so we made certain that, if nothing else, all the students in CENG 110 knew how to put together an accurate scientific graph. We also knew that some of the mathematical techniques were beyond the grasp of our first year students (e.g., linked O.D.E.s) and so we didn't worry about not teaching those skills. More basic skills (e.g., the trapezoidal rule) were, however, judged to be understandable to the students and were taught to them. And finally, we were gratified to see that the students have commented that the Excel instruction component of CENG 110 was successful in giving them an understanding of typical chemical engineering problems.

Although we do not as yet have enough data to provide a precise quantitative judgment, we do have anecdotal evidence that indicates teaching Excel to the first year students is useful. Instructors in subsequent courses report greater capabilities of their students and decreased time spent explaining possible solution methodologies. We believe that the learning of spreadsheet skills by the first year students will prove to be highly beneficial throughout the rest of the curriculum.

\footnotetext{
${ }^{1}$ William Josephson, K.C. Kwon and Nader Vahdat, "A Self-Assessment of Computer Science Education in a Chemical Engineering Curriculum”, Proceedings of the 2004 American Society for Engineering Education Annual Conference \& Exposition.

${ }^{2}$ Course Notes from U. of Leeds' CIVE 2601 Engineering Mathematics course, available on-line at http://www.efm.leeds.ac.uk/CIVE/CIVE2601

${ }^{3}$ Figures 1-4 in Vaclav Havlicek and Roman Cmejla, "Analysis of the Electrical Engineering Problems Using Computer Tools", Proceedings of International Conference on Engineering Education 2000.

${ }^{4}$ Example 4.3 from J.M Smith, H.C. Van Ness and M.M. Abbott, Introduction to Chemical Engineering Thermodynamics, $7^{\text {th }}$ ed., McGraw-Hill, 2005, page 132.

${ }^{5}$ This application of conversion versus reciprocal reaction rate curve is known as a "Levenspiel Plot" and is covered in the second chapter of H. Scott Fogler, Elements of Chemical Reaction Engineering, $4^{\text {th }}$ ed., Prentice Hall, 2005.

${ }^{6}$ This is adapted from Example 4-6, page 191 in Fogler. He used Polymath to generate the values of X and P which are generated by Excel in this paper.

${ }^{7}$ Euler's Method is covered in pages 111 and 112 of Carl E. Pearson, Numerical Methods in Engineering and Science, Van Nostrand Reinhold, 1986.

${ }^{8}$ The basics are covered in Chapter 4 of Richard M. Felder and Ronald W. Rousseau, Elementary Principles of Chemical Processes, $3^{\text {rd }}$ ed., John Wiley \& Sons, 2000.

${ }^{9}$ Adapted from the numerous examples in Chapter 3 of J.P. Holman, Heat Transfer, $8^{\text {th }}$ ed., McGraw-Hill, 1997.
} 